\title{
LARGE DEVIATIONS FOR MULTIDIMENSIONAL STATE-DEPENDENT SHOT-NOISE PROCESSES
}

\author{
AMARJIT BUDHIRAJA, ${ }^{*}$ University of North Carolina at Chapel Hill \\ PIERRE NYQUIST, ${ }^{* *}$ Brown University
}

\begin{abstract}
Shot-noise processes are used in applied probability to model a variety of physical systems in, for example, teletraffic theory, insurance and risk theory, and in the engineering sciences. In this paper we prove a large deviation principle for the sample-paths of a general class of multidimensional state-dependent Poisson shot-noise processes. The result covers previously known large deviation results for one-dimensional stateindependent shot-noise processes with light tails. We use the weak convergence approach to large deviations, which reduces the proof to establishing the appropriate convergence of certain controlled versions of the original processes together with relevant results on existence and uniqueness.
\end{abstract}

Keywords: Large deviations; Poisson shot-noise; Poisson random measure; variational representations

2010 Mathematics Subject Classification: Primary 60F10; 60G55

Secondary $60 \mathrm{~K} 30$

\section{Introduction}

The goal of this paper is to study large deviation results for a general family of multidimensional state-dependent shot-noise processes. Shot-noise processes provide a natural class of models for systems in which (some aspect of) the state of the system is determined by the arrival of shocks. A typical application is in the context of queueing systems, in which the arrival of customers can be interpreted as shocks and one is interested in, say, the current workload or cost incurred - due to performed work - by current and former customers. Another common area of application is insurance, the shocks being claims that arrive according to an underlying point process.

Due to their usefulness in describing various physical systems, shot-noise processes have been studied extensively, both theoretically as well as from the perspective of applications. For some general treatments of this class of processes and their properties, see [5], [7], [17], [18]. Vvarious asymptotic properties of shot-noise are found in [13] and [15], with [12], [16], and [19] dealing with heavy-tailed phenomena. An example of shot-noise processes in the queueing context is provided in [9], whereas [11] and [12] consider applications to insurance and risk theory. Another type of application is to storage processes, see, e.g. [2] and [14].

In this paper we are concerned with Poisson shot-noise processes, i.e. the underlying point process governing the arrivals of the shocks is a Poisson process. Large deviations for a family of

\footnotetext{
Received 25 July 2014.

* Postal address: Department of Statistics and Operations Research, University of North Carolina, Chapel Hill, NC 27599, USA. Email address: budhiraj@email.unc.edu

** Postal address: Division of Applied Mathematics, Brown University, Providence, RI 02912, USA.

Email address: pierre_nyquist@brown.edu
} 
Poisson shot-noise processes have been studied in [8] and the precise result therein is as follows. Let $N$ be a homogeneous Poisson process with unit intensity and let $Z_{1}, Z_{2}, \ldots$, be independent and identically distributed $\mathbb{X}$-valued random variables, each with distribution $v$ and independent of $N$. Here $\mathbb{X}$ is some locally compact Polish space. For a function $H: \mathbb{R}_{+} \times \mathbb{X} \rightarrow \mathbb{R}_{+}$, referred to as the shot shape, consider the Poisson shot-noise process $\{X(t) ; t \in[0, T]\}$, defined as

$$
X(t)=\sum_{n=1}^{N(t)} H\left(t-T_{n}, Z_{n}\right)
$$

where $T_{1}, T_{2}, \ldots$ are jump instants of $N$. Suppose that

$$
t \mapsto H(t, z) \text { is nondecreasing, càdlàg, and } H(0, z)=0 \text { for each } z \in \mathbb{X} \text {. }
$$

Let $h(z)=\lim _{t \rightarrow \infty} H(t, z)$. The function $h$ is referred to as the shot value for the shot-noise process $X$. Suppose that $h$ satisfies the following condition:

$$
\int_{\mathbb{X}} \mathrm{e}^{\vartheta h(z)} v(\mathrm{~d} z)<\infty \quad \text { for every } \vartheta \in \mathbb{R}
$$

For $\varepsilon>0$, let $X^{\varepsilon}(t)=\varepsilon X\left(\varepsilon^{-1} t\right), t \in[0, T]$. Paper [8] shows that $\left\{X^{\varepsilon}\right\}_{\varepsilon>0}$ satisfies a large deviation principle (LDP) in $D\left([0, T]: \mathbb{R}_{+}\right)$as $\varepsilon \rightarrow 0$, where $D\left([0, T]: \mathbb{R}_{+}\right)$denotes the space of càdlàg functions from $[0, T]$ to $\mathbb{R}_{+}$which is equipped with the usual Skorohod topology.

The goal of this paper is to study large deviation properties of general state-dependent multidimensional shot-noise processes. Such processes are natural models for systems where the impact of a shot depends on the current state of the system. In order to prove large deviation results, we use the fact that a Poisson shot-noise process can be represented as an integral with respect to a Poisson random measure. Using such representations, our work builds on certain variational formulae for functionals of a Poisson random measure [4] and their application to large deviations [3]. Rather than traditional large deviation techniques such as those used in [8], we use the weak convergence approach to large deviations. With the results of [3] and [4], this amounts to proving the appropriate convergence of certain controlled versions of the original process together with the necessary existence and uniqueness results. The main advantage of the weak convergence approach is that it avoids the discretization/approximation arguments and exponential estimates typically encountered in a large deviation analysis; see, e.g. [6]. Such approximation methods are used extensively in [8] and in general are difficult to implement for complex settings such as the state-dependent shot-noise processes considered here.

Large deviation results such as those considered in this paper can be used to determine the most likely path to rare events. In applications of shot-noise processes these results can thus be used to try and prevent unwanted behavior of the system in question. Moreover, large deviation results can be used to design efficient Monte Carlo algorithms. For an example of such simulations in the context of shot-noise processes, see [20]. Applications of our results to rare-event simulation problems will be studied in our future work.

We now introduce the multidimensional state-dependent shot-noise processes that will be studied in this paper. Let for each $\varepsilon>0, H_{\varepsilon}: \mathbb{R}_{+} \times \mathbb{X} \times \mathbb{R}_{+}^{d} \rightarrow \mathbb{R}_{+}^{d}$ be a measurable function and consider the stochastic process $\tilde{X}^{\varepsilon}$ given as the solution of the equation:

$$
\tilde{X}^{\varepsilon}(t)=\sum_{n=1}^{N(t)} H_{\varepsilon}\left(t-T_{n}, Z_{n}, \tilde{X}^{\varepsilon}\left(T_{n}-\right)\right), \quad t \geq 0 .
$$


Let $X^{\varepsilon}(t)=\varepsilon \tilde{X}^{\varepsilon}\left(\varepsilon^{-1} t\right)$. We will give a sufficient condition on the collection of maps $\left\{H_{\varepsilon}\right\}_{\varepsilon>0}$ under which $\left\{X^{\varepsilon}\right\}_{\varepsilon>0}$ satisfies an LDP in $D\left([0, T]: \mathbb{R}_{+}^{d}\right)$. It will be convenient to work with the following, equivalent in law, representation for $X^{\varepsilon}$. Let $\boldsymbol{n}_{\varepsilon}$ be a Poisson random measure $(\mathrm{PRM})$ on $\mathbb{X}_{T}=[0, T] \times \mathbb{X}$ with intensity $\varepsilon^{-1} \nu_{T}=\varepsilon^{-1} \lambda \otimes \nu$, where $\lambda$ is the Lebesgue measure on $[0, T]$. Let $X^{\varepsilon}$ solve

$$
X^{\varepsilon}(t)=\varepsilon \int_{\mathbb{X}_{t}} H_{\varepsilon}\left(\varepsilon^{-1}(t-s), z, \varepsilon^{-1} X^{\varepsilon}(s-)\right) n_{\varepsilon}(\mathrm{d} s \mathrm{~d} z), \quad t \in[0, T],
$$

where $\mathbb{X}_{t}=[0, t] \times \mathbb{X}$ for $t \in[0, T]$. It is easy to check that $X^{\varepsilon}$ defined in $(1.5)$ and $\varepsilon \tilde{X}^{\varepsilon}\left(\varepsilon^{-1} \cdot\right)$, where $\tilde{X}^{\varepsilon}$ is as in (1.4), have the same distribution. We will in fact consider a more general setting in that we will study the collection $\left\{X^{\varepsilon}\right\}_{\varepsilon>0}$ given as the solution of (1.5), where the measure $v$ describing the intensity of $\boldsymbol{n}_{\varepsilon}$ is a general $\sigma$-finite measure on $(\mathbb{X}, \mathscr{B}(\mathbb{X}))$. This allows for a nonintegrable number of shocks on a bounded time interval. One can formulate general sufficient conditions under which (1.5) has a unique pathwise solution. We will instead take unique solvability of the equation as one of our basic assumptions (see Condition 2.1). In Section 2.1 we introduce our assumptions (Conditions 2.1 and 2.2) on $H_{\varepsilon}$. Our main result (Theorem 2.2) shows that under these conditions, $X^{\varepsilon}$ given as the solution of the stochastic integral equation (2.1) satisfies an LDP in $D\left([0, T]: \mathbb{R}_{+}^{d}\right)$ as $\varepsilon \rightarrow 0$. The LDP established in [8] is an immediate consequence of Theorem 2.2.

The rest of the paper is organized as follows. Section 3 contains the proof of well-posedness of an ordinary differential equation (ODE) associated with the asymptotics of controlled analogues of (1.5) (Theorem 2.1). In Section 4 we recall a result from [4] that gives a general sufficient condition (Condition 4.1) for an LDP to hold for measurable functionals of a PRM. Theorem 2.2 is proved by verifying this sufficient condition. Condition 4.1(a) is verified in Section 5.1 while Condition 4.1(b) is considered in Section 5.2.

The following notation will be used. The space of probability measures on a Polish space $\mathbb{S}$, equipped with the topology of weak convergence, will be denoted by $\mathcal{P}(\mathbb{S})$. For a function $f:[0, T] \rightarrow \mathbb{R}^{k}$, set $\|f\|_{*, t}=\sup _{0 \leq s \leq t}\|f(s)\|, t \in[0, T]$. For $\vartheta \in \mathcal{P}(\mathbb{S})$ and a $\vartheta$-integrable $f$ on $\mathbb{S}$, we denote $\int_{\mathbb{S}} f(x) \vartheta(\mathrm{d} x)$ as $\langle f, \vartheta\rangle$. The Borel $\sigma$-field on a Polish space $\mathbb{S}$ will be denoted as $\mathscr{B}(\mathbb{S})$. The space of functions that are right-continuous with left limits from $[0, \infty)$ (respectively $[0, T])$ to $\mathbb{S}$ will be denoted as $D([0, \infty): \mathbb{S}$ ) (respectively $D([0, T]: \mathbb{S})$ ) and are equipped with the usual Skorohod topology. For a bounded function $f$ from $\mathbb{S}$ to $\mathbb{R}$, we denote $\|f\|_{\infty}=\sup _{x \in \mathbb{S}}|f(x)|$. Convergence of a sequence $\left\{X_{n}\right\}$ of $\mathbb{S}$-valued random variables in distribution to $X$ will be written as $X_{n} \stackrel{\mathrm{D}}{\rightarrow} X$.

For a $\sigma$-finite measure $v$ on a Polish space $\mathbb{S}, \mathcal{L}_{\mathbb{R}^{k}}^{p}(\mathbb{S}, v)$ will denote the space of $p$-integrable functions with respect to $v$, from $\mathbb{S}$ to $\mathbb{R}^{k}$. When $k=1$, we will merely write $\mathcal{L}^{p}(\mathbb{S}, v)$ or $\mathcal{L}^{p}(v)$. We will usually denote by $\kappa, \kappa_{1}, \kappa_{2}, \ldots$, the constants that appear in various estimates within a proof. The values of these constants may change from one proof to another.

\section{Main result}

Our basic collection of stochastic processes $\left\{X^{\varepsilon}\right\}_{\varepsilon>0}$ is given in terms of PRMs $\left\{\boldsymbol{n}_{\varepsilon}\right\}_{\varepsilon>0}$. We would like all these PRMs to be defined on a common probability space. For this, the following construction will be useful. Let $\mathbb{Y}=\mathbb{X} \times[0, \infty)$ and $\mathbb{Y}_{T}=[0, T] \times \mathbb{Y}$. For a locally compact Polish space $\mathbb{Z}$, let $\mathcal{M}_{\mathrm{FC}}(\mathbb{Z})$ be the space of all measures $v$ on $(\mathbb{Z}, \mathbb{B}(\mathbb{Z}))$ such that $v(K)<\infty$ for every compact $K$ in $\mathbb{Z}$. This space is equipped with the usual topology of vague convergence. Let $\bar{M}=\mathcal{M}_{\mathrm{FC}}\left(\mathbb{Y}_{T}\right)$ and let $\overline{\mathbb{P}}$ be the unique probability measure on $(\overline{\mathrm{M}}, \mathscr{B}(\overline{\mathrm{M}}))$ under which the 
canonical map, $\bar{N}: \bar{M} \rightarrow \bar{M}, \bar{N}(m)=m$, is a PRM with intensity measure $\bar{v}_{T}=\lambda \otimes v \otimes \lambda_{\infty}$, where $\lambda_{\infty}$ is the Lebesgue measure on $[0, \infty)$. The corresponding expectation operator will be denoted by $\overline{\mathbb{E}}$. Let $\mathcal{F}_{t}=\sigma\{\bar{N}((0, s] \times A): 0 \leq s \leq t, A \in \mathscr{B}(\mathbb{Y})\}$, and let $\overline{\mathcal{F}}_{t}$ denote the completion under $\overline{\mathbb{P}}$. We denote by $\overline{\mathcal{P}}$ the predictable $\sigma$-field on $[0, T] \times \overline{\mathrm{M}}$ with the filtration $\left\{\overline{\mathcal{F}}_{t}: 0 \leq t \leq T\right\}$ on $(\bar{M}, \mathscr{B}(\bar{M}))$.

For $\varepsilon>0$, let $N^{\varepsilon^{-1}}$ be a counting process on $\mathbb{X}_{T}$ defined as

$$
N^{\varepsilon^{-1}}((0, t] \times U)=\int_{(0, t] \times U \times \mathbb{R}_{+}} \mathbf{1}_{\left[0, \varepsilon^{-1}\right]}(r) \bar{N}(\mathrm{~d} s \mathrm{~d} x \mathrm{~d} r), \quad t \in[0, T], U \in \mathscr{B}(\mathbb{X}) .
$$

Clearly $N^{\varepsilon^{-1}}$ has the same distribution as $\boldsymbol{n}_{\varepsilon}$, i.e. it is a PRM on $\mathbb{X}_{T}=[0, T] \times \mathbb{X}$ with intensity $\varepsilon^{-1} \lambda \otimes \nu$.

Next we introduce our assumptions and present the main result.

\subsection{Assumptions}

Our first assumption is on the unique solvability of (1.5). Note that $N^{\varepsilon^{-1}}$ is a $\mathbb{M}$-valued random variable, where $\mathbb{M}=\mathcal{M}_{\mathrm{FC}}\left(\mathbb{X}_{T}\right)$.

Condition 2.1. For each $\varepsilon>0$, there is a measurable map $g^{\varepsilon}: \mathbb{M} \rightarrow D\left([0, T]: \mathbb{R}_{+}^{d}\right)$ such that for any probability space $(\tilde{\Omega}, \tilde{\mathcal{F}}, \tilde{P})$ on which is given a PRM $\tilde{n}_{\varepsilon}$ on $\mathbb{X}_{T}$ with intensity measure $\varepsilon^{-1} v_{T}, X^{\varepsilon}=g^{\varepsilon}\left(\varepsilon \tilde{\boldsymbol{n}}_{\varepsilon}\right)$ is a $\tilde{\mathcal{F}}_{t}=\sigma\left\{\tilde{\boldsymbol{n}}_{\varepsilon}(B \times[0, s]), s \leq t, B \in \mathscr{B}(\mathbb{X}), v(B)<\infty\right\}$ adapted càdlàg process that is the unique solution of the stochastic integral equation (1.5).

Condition 2.1 is satisfied quite generally. For example, if $v$ is a finite measure, the unique solvability is immediate from a recursive construction of a solution of (1.5) from one jump to the next. For more general $v$, Condition 2.1 will hold under suitable Lipschitz and growth assumptions on $H_{\varepsilon}$ (cf. [10, Theorem III.2.3.2]). The condition, in particular, says that $X^{\varepsilon}=g^{\varepsilon}\left(\varepsilon N^{\varepsilon^{-1}}\right)$ is the unique solution of

$$
X^{\varepsilon}(t)=\varepsilon \int_{\mathbb{X}_{t}} H_{\varepsilon}\left(\varepsilon^{-1}(t-s), z, \varepsilon^{-1} X^{\varepsilon}(s-)\right) N^{\varepsilon^{-1}}(\mathrm{~d} s \mathrm{~d} z), \quad t \in[0, T]
$$

on $(\overline{\mathbb{M}}, \mathscr{B}(\bar{M}), \overline{\mathbb{P}})$. For the rest of this paper $X^{\varepsilon}$ will denote the solution of $(2.1)$.

Next we introduce our second main assumption on the family $\left\{H_{\varepsilon}\right\}_{\varepsilon>0}$. We denote by $\mathcal{L}_{\exp }$ the family of all measurable functions $r: \mathbb{X} \rightarrow \mathbb{R}_{+}$such that whenever $A \in \mathscr{B}(\mathbb{X})$ is such that $v(A)<\infty$,

$$
\int_{A} \mathrm{e}^{\vartheta r(z)} v(\mathrm{~d} z)<\infty \quad \text { for all } \vartheta \in \mathbb{R}
$$

Note that if $v$ is a probability measure, this condition merely says that $r(Z)$ has an everywhere finite moment generating function, where $Z$ is a random variable with probability distribution $\nu$.

Condition 2.2. There are measurable functions $\bar{H}$ and $R_{\varepsilon}$ from $\mathbb{R}_{+} \times \mathbb{X} \times \mathbb{R}_{+}^{d}$ to $\mathbb{R}_{+}^{d}$ and $\mathbb{R}^{d}$, respectively; $\varsigma_{\varepsilon}, \varsigma$ from $\mathbb{X}$ to $\mathbb{R}_{+} ;$and $h$ from $\mathbb{X} \times \mathbb{R}_{+}^{d}$ to $\mathbb{R}_{+}^{d}$ such that the following hold.

(a) For $\varepsilon>0$ and $(t, z, x) \in \mathbb{R}_{+} \times \mathbb{X} \times \mathbb{R}_{+}^{d}$,

$$
H_{\varepsilon}(t, z, x)=\bar{H}(t, z, \varepsilon x)+R_{\varepsilon}(t, z, \varepsilon x) .
$$

(b) For $\varepsilon>0$ and $(z, x) \in \mathbb{X} \times \mathbb{R}_{+}^{d}$,

$$
\sup _{t \geq 0}\left\|R_{\varepsilon}(t, z, x)\right\| \leq \varsigma_{\varepsilon}(z)(\|x\|+1) .
$$


(c) $\varsigma_{\varepsilon} \leq \varsigma, \varsigma \in \mathcal{L}_{\exp } \cap \mathcal{L}^{1}(v)$ and for $v$ almost everywhere (a.e.) $z \in \mathbb{X}, \varsigma_{\varepsilon}(z) \rightarrow 0$ as $\varepsilon \rightarrow 0$.

(d) For $(z, x) \in \mathbb{X} \times \mathbb{R}_{+}^{d}, t \mapsto \bar{H}(t, z, x)$ is càdlàg and nondecreasing (coordinatewise) and $\bar{H}(0, z, x)=0$.

(e) For every $z \in \mathbb{X}$ and $m>0, \sup _{\|x\| \leq m} \mid \bar{H}(t, z, x)-h(z, x) \| \rightarrow 0$ as $t \rightarrow \infty$.

(f) For some $L_{h} \in \mathcal{L}_{\exp } \cap \mathcal{L}^{1}(v)$,

$$
\left\|h(z, x)-h\left(z, x^{\prime}\right)\right\| \leq L_{h}(z)\left\|x-x^{\prime}\right\| \text { for all } x, x^{\prime} \in \mathbb{R}_{+}^{d}, z \in \mathbb{X}
$$

(g) For some $M_{h} \in \mathcal{L}_{\exp } \cap \mathcal{L}^{1}(v)$

$$
\|h(z, x)\| \leq M_{h}(z)(1+\|x\|) \text { for all } x \in \mathbb{R}_{+}^{d}, z \in \mathbb{X} \text {. }
$$

The setting considered in [8] corresponds to the case where $H_{\varepsilon}(t, z, x)$ is independent of $\varepsilon$ and $x$; in particular $R_{\varepsilon} \equiv 0$. Conditions 2.1 and 2.2 will be standing assumptions for this paper and will not be explicitly mentioned in the statements of results.

\subsection{Controlled ODEs}

In this section we will consider an ODE that arises in the asymptotic analysis of the controlled analogues of (2.1). Define $l:[0, \infty) \rightarrow[0, \infty)$ by

$$
l(r)=r \log r-r+1, \quad r \in[0, \infty)
$$

For $g: \mathbb{X}_{T} \rightarrow[0, \infty)$, let

$$
\begin{gathered}
L_{T}(g)=\int_{\mathbb{X}_{T}} l(g(t, z)) \nu_{T}(\mathrm{~d} t \mathrm{~d} z), \\
S^{n}=\left\{g: \mathbb{X}_{T} \rightarrow[0, \infty): L_{T}(g) \leq n\right\} .
\end{gathered}
$$

A function $g \in S^{n}$ can be identified with a measure $\nu_{T}^{g} \in \mathbb{M}$ defined by

$$
v_{T}^{g}(A)=\int_{A} g(s, x) \nu_{T}(\mathrm{~d} s \mathrm{~d} x), \quad A \in \mathscr{B}\left(\mathbb{X}_{T}\right)
$$

This identification induces a topology on $S^{n}$ under which $S^{n}$ is a compact space; see [3] for a proof. Let $S=\bigcup_{n \geq 1} S^{n}$. For $g \in S$ consider the integral equation

$$
\xi(t)=\int_{\mathbb{X}_{t}} h(z, \xi(s)) g(s, z) v(\mathrm{~d} z) \mathrm{d} s, \quad t \in[0, T]
$$

The following result says that the above integral equation has a unique solution for every $g \in S$; a proof is given in Section 3.

Theorem 2.1. For every $g \in S$ there is a unique $\xi \in C\left([0, T]: \mathbb{R}_{+}^{d}\right)$ that solves (2.3). 


\subsection{The LDP}

We are now ready to present our main result. Given $g \in S$ denote by $\xi^{g}$ the unique solution of (2.3). Define $I: D\left([0, T]: \mathbb{R}_{+}^{d}\right) \rightarrow[0, \infty]$ as

$$
I(\phi)=\inf _{g \in S: \phi=\xi^{g}}\left\{L_{T}(g)\right\},
$$

where infimum over an empty set is taken to be $\infty$. In particular, this says that $I(\phi)=\infty$ for all $\phi \in D\left([0, T]: \mathbb{R}_{+}^{d}\right) \backslash C\left([0, T]: \mathbb{R}_{+}^{d}\right)$. The following is our main result.

Theorem 2.2. It holds that $I$ is a rate function and the collection $\left\{X^{\varepsilon}\right\}_{\varepsilon>0}$ satisfies an LDP in $D\left([0, T]: \mathbb{R}_{+}^{d}\right)$ with rate function $I$ as $\varepsilon \rightarrow 0$.

Remark 2.1. The LDP for the scalar state-independent case established in [8, Proposition 3.1] is an immediate consequence of Theorem 2.2. To see this, note that when $d=1$ and $H_{\varepsilon}(t, z, x) \equiv H(t, z)$, where $H$ is as introduced in (1.1), Condition 2.1 holds trivially. Furthermore, under the assumptions made in [8] (specifically, (1.2)), Conditions 2.2(a)-(f) are immediate and $h(z, x) \equiv h(z)$. Finally, the requirement in (1.3), and since $v$ is a probability measure, implies that Condition 2.2(g) holds as well in this state-independent case.

\section{Proof of Theorem 2.1}

In this section we prove Theorem 2.1. We start with the following two lemmas which will be used several times in this paper. The proof of the first lemma is standard and is omitted.

Lemma 3.1. (a) For $a, b \in(0, \infty)$ and $\sigma \in[1, \infty), a b \leq \mathrm{e}^{\sigma a}+(1 / \sigma) l(b)$.

(b) For every $\beta>0$, there exist $\varrho_{1}(\beta), \varrho_{2}(\beta) \in(0, \infty)$ such that $\varrho_{1}(\beta), \varrho_{2}(\beta) \rightarrow 0$ as $\beta \rightarrow \infty$, and

$$
|x-1| \leq \varrho_{1}(\beta) l(x) \text { for }|x-1| \geq \beta, x \geq 0, \quad x \leq \varrho_{2}(\beta) l(x) \text { for } x \geq \beta>1 .
$$

Lemma 3.2. Let $\vartheta \in \mathcal{L}_{\exp } \cap \mathcal{L}^{1}(v)$. For every $\delta>0$ and $n \in \mathbb{N}$, there exists $c(\delta, n, \vartheta) \in$ $(0, \infty)$ such that for all $\tilde{\vartheta}: \mathbb{X} \rightarrow \mathbb{R}_{+}$such that $\tilde{\vartheta} \leq \vartheta$, all measurable maps $f:[0, T] \rightarrow \mathbb{R}_{+}$ and $0 \leq s \leq t \leq T$,

$$
\begin{aligned}
& \sup _{g \in S^{n}} \int_{(s, t] \times \mathbb{X}} f(u) \tilde{\vartheta}(z) g(u, z) v(\mathrm{~d} z) \mathrm{d} u \\
& \quad \leq c(\delta, n, \vartheta)\left(\int_{\mathbb{X}} \tilde{\vartheta}(z) v(\mathrm{~d} z)\right)\left(\int_{S}^{t} f(u) \mathrm{d} u\right)+\delta|f|_{*, t} .
\end{aligned}
$$

Proof. Let $f:[0, T] \rightarrow \mathbb{R}_{+}, g \in S^{n}$, and $\tilde{\vartheta}, \vartheta$ be as in the statement of the lemma. Then, for each $m>0$,

$$
\int_{(s, t] \times \mathbb{X}} f(u) \tilde{\vartheta}(z) g(u, z) v(\mathrm{~d} z) \mathrm{d} u=T_{1}(m)+T_{2}(m)
$$

where

$$
T_{1}(m)=\int_{(s, t] \times\{\vartheta \leq m\}} f(u) \tilde{\vartheta}(z) g(u, z) v(\mathrm{~d} z) \mathrm{d} u
$$

and

$$
T_{2}(m)=\int_{(s, t] \times\{\vartheta>m\}} f(u) \tilde{\vartheta}(z) g(u, z) v(\mathrm{~d} z) \mathrm{d} u .
$$


Using Lemma 3.1(a), we can estimate $T_{2}(m)$, for each $k \geq 1$, as

$$
T_{2}(m) \leq|f|_{*, t}\left(T \int_{\{\vartheta>m\}} \mathrm{e}^{k \vartheta(z)} v(\mathrm{~d} z)+\frac{n}{k}\right)
$$

For each $\beta>1$, define the sets $E_{1}(m, \beta)$ and $E_{2}(m, \beta)$ by

$$
\begin{aligned}
& E_{1}(m, \beta)=\{(u, z) \in(s, t] \times \mathbb{X}: \vartheta(z) \leq m \text { and } g(s, z) \leq \beta\} \\
& E_{2}(m, \beta)=\{(u, z) \in(s, t] \times \mathbb{X}: \vartheta(z) \leq m \text { and } g(s, z)>\beta\}
\end{aligned}
$$

Then $T_{1}(m)$ can be estimated as

$$
T_{1}(m) \leq T_{3}(m, \beta)+T_{4}(m, \beta)
$$

where

$$
\begin{aligned}
& T_{3}(m, \beta)=\int_{E_{1}(m, \beta)} f(u) \tilde{\vartheta}(z) g(u, z) v(\mathrm{~d} z) \mathrm{d} u \\
& T_{4}(m, \beta)=\int_{E_{2}(m, \beta)} f(u) \tilde{\vartheta}(z) g(u, z) v(\mathrm{~d} z) \mathrm{d} u .
\end{aligned}
$$

Using Lemma 3.1(b),

$$
T_{3}(m, \beta)+T_{4}(m, \beta) \leq \beta\left(\int_{\mathbb{X}} \tilde{\vartheta}(z) v(\mathrm{~d} z)\right)\left(\int_{s}^{t} f(u) \mathrm{d} u\right)+\varrho_{2}(\beta) m n|f|_{*, t} .
$$

Combining the estimates for $T_{1}(m)$ and $T_{2}(m)$, the left-hand side of (3.1) can be bounded by

$$
\beta\left(\int_{\mathbb{X}} \tilde{\vartheta}(z) v(\mathrm{~d} z)\right)\left(\int_{s}^{t} f(u) \mathrm{d} u\right)+|f|_{*, t}\left(\varrho_{2}(\beta) m n+T \int_{\{\vartheta>m\}} \mathrm{e}^{k \vartheta(z)} v(\mathrm{~d} z)+\frac{n}{k}\right) .
$$

Now, given $\delta>0$, choose $k>1$ such that $n / k<\delta / 3$. Next, since $\vartheta \in \mathcal{L}_{\exp } \cap \mathcal{L}^{1}(v)$, it is possible to choose $m>0$ such that $T \int_{\{\vartheta>m\}} \mathrm{e}^{k \vartheta(z)} \nu(\mathrm{d} z)<\delta / 3$. Finally, using Lemma 3.1(b), choose $\beta>1$ such that $\varrho_{2}(\beta) m n<\delta / 3$. The result now follows on taking $c(\delta, n, \vartheta)=\beta$.

Proof of Theorem 2.1. We will use Banach's fixed point theorem. Fix $n \in \mathbb{N}$ and $g \in S^{n}$. Define for $r>0, T^{r}: C\left([0, r]: \mathbb{R}_{+}^{d}\right) \rightarrow C\left([0, r]: \mathbb{R}_{+}^{d}\right)$ as

$$
T^{r}(\phi)(t)=\phi(0)+\int_{\mathbb{X}_{t}} h(z, \phi(s)) g(s, z) v(\mathrm{~d} z) \mathrm{d} s, \quad t \in[0, r], \phi \in C\left([0, r]: \mathbb{R}_{+}^{d}\right)
$$

Note that the right-hand side indeed defines an element of $C\left([0, r]: \mathbb{R}_{+}^{d}\right)$ since by Lemma 3.2, for $\delta>0$ and $0 \leq s<t<r$,

$$
\begin{aligned}
\int_{(s, t] \times \mathbb{X}}\|h(z, \phi(u))\| g(s, u) v(\mathrm{~d} z) \mathrm{d} u \\
\leq\left(1+\|\phi\|_{*, r}\right)\left(c\left(\delta, n, M_{h}\right)(t-s) \int_{\mathbb{X}} M_{h}(z) v(\mathrm{~d} z)+\delta\right) .
\end{aligned}
$$


We will now argue that for $r$ small enough $T^{r}$ is a contraction. Note that for $\phi, \tilde{\phi} \in C([0, r]$ : $\left.\mathbb{R}_{+}^{d}\right)$, with $\phi(0)=\tilde{\phi}(0)$,

$$
\begin{aligned}
\left\|T^{r}(\phi)-T^{r}(\tilde{\phi})\right\|_{*, r} & \leq \int_{\mathbb{X}_{r}}\|h(z, \phi(s))-h(z, \tilde{\phi}(s))\| g(s, z) v(\mathrm{~d} z) \mathrm{d} s \\
& \leq\|\phi-\tilde{\phi}\|_{*, r} \int_{\mathbb{X}_{r}} L_{h}(z) g(s, z) v(\mathrm{~d} z) \mathrm{d} s .
\end{aligned}
$$

Using Lemma 3.2 again and our assumption on $L_{h}$, we have $\int_{\mathbb{X}_{T}} L_{h}(z) g(s, z) v(\mathrm{~d} z) \mathrm{d} s<\infty$. Thus, for sufficiently small $r, \int_{\mathbb{X}_{r}} L_{h}(z) g(s, z) v(\mathrm{~d} z) \mathrm{d} s<1$ and consequently $T^{r}$ is a contraction and so by Banach's fixed point theorem has a unique fixed point. This shows that there is a unique solution to (2.3) for all $t \in[0, r]$. The result now follows by a recursive argument.

\section{A general sufficient condition for the LDP}

We now present a result from [4] which will be a key ingredient in our proofs. We begin with some notation. Let $\overline{\mathcal{A}}$ be the class of all $(\overline{\mathcal{P}} \otimes \mathscr{B}(\mathbb{X})) / \mathscr{B}[0, \infty)$-measurable maps $\varphi: \mathbb{X}_{T} \times \overline{\mathbb{M}} \rightarrow[0, \infty)$; as is common, we frequently suppress in the notation the dependence of $\varphi$ on elements in (the probability space) $\bar{M}$. For $\varphi \in \overline{\mathcal{A}}$, define a counting process $N^{\varphi}$ on $\mathbb{X}_{T}$ by

$$
N^{\varphi}((0, t] \times U)=\int_{(0, t] \times U \times \mathbb{R}_{+}} \mathbf{1}_{[0, \varphi(s, z)]}(r) \bar{N}(\mathrm{~d} s \mathrm{~d} z \mathrm{~d} r), \quad t \in[0, T], U \in \mathscr{B}(\mathbb{X}) .
$$

The counting process $N^{\varphi}$ can be interpreted as a controlled random measure, with $\varphi$ playing the role of the control which selects the intensity for the points at location $x$ and time $s$ in a possibly random but nonanticipating way. Let

$$
U^{n}=\left\{\varphi \in \overline{\mathcal{A}}:(s, z) \mapsto \varphi(s, z, \omega) \in S^{n}, \overline{\mathbb{P}} \text { a.e. } \omega\right\}
$$

Elements of $U^{n}$ will be regarded as $S^{n}$-valued random variables where the topology on the latter space is as introduced below (2.2). Let $\left\{K_{m} \subset \mathbb{X}, m=1,2, \ldots\right\}$ be an increasing sequence of compact sets such that $\bigcup_{m=1}^{\infty} K_{m}=\mathbb{X}$. For each $m$, let

$$
\begin{gathered}
\overline{\mathcal{A}}_{b, m}=\left\{\varphi \in \overline{\mathcal{A}}: \text { for all }(t, \omega) \in[0, T] \times \overline{\mathbb{M}}, m \geq \varphi(t, x, \omega) \geq \frac{1}{m} \text { if } x \in K_{m}\right. \\
\text { and } \left.\varphi(t, x, \omega)=1 \text { if } x \in K_{m}^{c}\right\}
\end{gathered}
$$

and let $\overline{\mathscr{A}}_{b}=\bigcup_{m=1}^{\infty} \overline{\mathscr{A}}_{b, m}$. Define $\tilde{U}^{n}=U^{n} \cap \overline{\mathscr{A}}_{b}$.

Let $\mathbb{U}$ be a Polish space. The following condition is a slight modification of a condition introduced in [4, Section 4] to establish a large deviation result; see [3, Section 2.2].

Condition 4.1. There exist measurable maps $g^{0}, g^{\varepsilon}, \varepsilon>0$, from $\mathbb{M}$ to $\mathbb{U}$ such that the following hold.

(a) For $n \in \mathbb{N}$, let $g_{m}, g \in S^{n}$ be such that $g_{m} \rightarrow g$ as $m \rightarrow \infty$. Then

$$
g^{0}\left(\nu_{T}^{g_{m}}\right) \rightarrow g^{0}\left(v_{T}^{g}\right)
$$


(b) For $n \in \mathbb{N}$, let $\varphi_{\varepsilon}, \varphi \in \tilde{U}^{n}$ be such that $\varphi_{\varepsilon}$ converges in distribution to $\varphi$ as $\varepsilon \rightarrow 0$. Then

$$
g^{\varepsilon}\left(\varepsilon N^{\varepsilon^{-1}} \varphi_{\varepsilon}\right) \stackrel{\mathrm{D}}{\rightarrow} g^{0}\left(\nu_{T}^{\varphi}\right)
$$

For $\phi \in \mathbb{U}$, define $\mathbb{S}_{\phi}=\left\{g \in S: \phi=g^{0}\left(v_{T}^{g}\right)\right\}$. Let $I: \mathbb{U} \rightarrow[0, \infty]$ be defined by

$$
I(\phi)=\inf _{g \in \mathbb{S}_{\phi}}\left\{L_{T}(g)\right\}, \quad \phi \in \mathbb{U} .
$$

By convention, $I(\phi)=\infty$ if $\mathbb{S}_{\phi}=\varnothing$.

The following theorem is a slight extension of [4, Theorem 4.2]. For a proof, we refer the reader to [3, Appendix].

Theorem 4.1. For $\varepsilon>0$, let $Z^{\varepsilon}$ be defined by $Z^{\varepsilon}=g^{\varepsilon}\left(\varepsilon N^{\varepsilon^{-1}}\right)$. If Condition 4.1 holds then I defined as in (4.1) is a rate function on $\mathbb{U}$ and the family $\left\{Z^{\varepsilon}\right\}_{\varepsilon>0}$ satisfies an LDP with rate function $I$.

\section{Proof of Theorem 2.2}

In order to prove Theorem 2.2 we will apply Theorem 4.1 with $\mathbb{U}=D\left([0, T]: \mathbb{R}_{+}^{d}\right), g^{\varepsilon}$ as introduced in Condition 2.1, and $g^{0}: \mathbb{M} \rightarrow C\left([0, T]: \mathbb{R}_{+}^{d}\right)$ defined as follows. Let $g^{0}(m)=\xi^{g}$ if $m=v_{T}^{g}$ for some $g \in S$, where $\xi^{g}$ is as introduced above (2.4). For all other $m \in \mathbb{M}$ we set $g^{0}(m)=0$. It suffices to show that Condition 4.1 is satisfied with this choice of $g^{\varepsilon}$ and $g^{0}$. In Section 5.1 we will verify Condition 4.1(a) and Section 5.2 is devoted to the verification of Condition 4.1(b).

\subsection{Verification of Condition 4.1(a)}

The following is the main result of this section.

Proposition 5.1. Let $n \in \mathbb{N}$ and $g_{k}, g \in S^{n}, k \geq 1$ be such that $g_{k} \rightarrow g$. Then $\xi^{g_{k}} \rightarrow \xi^{g}$ in $C\left([0, T]: \mathbb{R}_{+}^{d}\right)$.

The following lemma will be useful in proving the proposition.

Lemma 5.1. Let $f_{k}, f \in D\left([0, T]: \mathbb{R}_{+}^{d}\right), k \geq 1$, be such that $\left\|f_{k}-f\right\|_{*, T} \rightarrow 0$ as $k \rightarrow \infty$. Also let $n \in \mathbb{N}$ and $g_{k}, g \in S^{n}$ be such that $g_{k} \rightarrow g$. Then letting

$$
\tilde{f}_{k}(t)=\int_{\mathbb{X}_{t}} h\left(z, f_{k}(s)\right) g_{k}(s, z) v(\mathrm{~d} z) \mathrm{d} s, \quad \tilde{f}(t)=\int_{\mathbb{X}_{t}} h(z, f(s)) g(s, z) v(\mathrm{~d} z) \mathrm{d} s,
$$

$\tilde{f}_{k}(t) \rightarrow \tilde{f}(t)$ as $k \rightarrow \infty$ for every $t \in[0, T]$.

Proof. Note that

$$
\int_{\mathbb{X}_{t}} h\left(z, f_{k}(s)\right) g_{k}(s, z) v(\mathrm{~d} z) \mathrm{d} s-\int_{\mathbb{X}_{t}} h(z, f(s)) g(s, z) v(\mathrm{~d} z) \mathrm{d} s=T_{1}^{k}(t)+T_{2}^{k}(t),
$$

where

$$
T_{1}^{k}(t)=\int_{\mathbb{X}_{t}}\left[h\left(z, f_{k}(s)\right)-h(z, f(s))\right] g_{k}(s, z) v(\mathrm{~d} z) \mathrm{d} s
$$

and

$$
T_{2}^{k}(t)=\int_{\mathbb{X}_{t}} h(z, f(s))\left[g_{k}(s, z)-g(s, z)\right] v(\mathrm{~d} z) \mathrm{d} s
$$


Noting that

$$
\left\|T_{1}^{k}\right\|_{*, T} \leq\left\|f_{k}-f\right\|_{*, T} \int_{\mathbb{X}_{T}} L_{h}(z) g_{k}(s, z) v(\mathrm{~d} z) \mathrm{d} s
$$

and $\sup _{k} \int_{\mathbb{X}_{T}} L_{h}(z) g_{k}(s, z) \nu(\mathrm{d} z) \mathrm{d} s<\infty$ from Lemma 3.2, we see that $\left\|T_{1}^{k}\right\|_{*, T} \rightarrow 0$ as $k \rightarrow$ $\infty$. Consider now $T_{2}^{k}$. We first claim that for every $\varepsilon>0$ there is a compact $K \subset \mathbb{X}$ such that

$$
\sup _{\psi \in S^{n}} \int_{[0, T] \times K^{c}} M_{h}(z) \psi(s, z) v(\mathrm{~d} z) \mathrm{d} s<\varepsilon .
$$

To see the claim, let $\left\{K_{\gamma}\right\}_{\gamma \in \mathbb{N}}$ be a sequence of compact subsets of $\mathbb{X}$ such that $K_{\gamma} \uparrow \mathbb{X}$ as $\gamma \rightarrow \infty$. Since $M_{h} \in \mathcal{L}^{1}(\nu)$,

$$
\int_{K_{\gamma}^{c}} M_{h}(z) v(\mathrm{~d} z) \rightarrow 0 \quad \text { as } \gamma \rightarrow \infty
$$

Also, from Lemma 3.2, with $f(u) \equiv 1, \tilde{\vartheta}(z)=1_{K_{\gamma}^{c}}(z) M_{h}(z)$, and $\vartheta(z)=M_{h}(z)$, we have, for every $\delta>0$,

$$
\sup _{\psi \in S^{n}} \int_{[0, T] \times K_{\gamma}^{c}} M_{h}(z) \psi(s, z) v(\mathrm{~d} z) \mathrm{d} s \leq c\left(\delta, n, M_{h}\right) T \int_{K_{\gamma}^{c}} M_{h}(z) v(\mathrm{~d} z)+\delta .
$$

The claim now follows on combining the above two displayed equations. Using (5.2), for a fixed $\varepsilon>0$ choose a compact $K \subset \mathbb{X}$ such that

$$
T_{2}^{k}(t)=\int_{\mathbb{X}_{t}} h(z, f(s)) \mathbf{1}_{K}(z)\left[g_{k}(s, z)-g(s, z)\right] v(\mathrm{~d} z) \mathrm{d} s+T_{3}^{k}(t)
$$

and $\sup _{k \geq 1}\left\|T_{3}^{k}\right\|_{*, T} \leq \varepsilon$. Next, for $\rho>0$ write

$$
\int_{\mathbb{X}_{t}} h(z, f(s)) \mathbf{1}_{K}(z)\left[g_{k}(s, z)-g(s, z)\right] v(\mathrm{~d} z) \mathrm{d} s=T_{4, \rho}^{k}(t)+T_{5, \rho}^{k}(t),
$$

where

$$
T_{4, \rho}^{k}(t)=\int_{[0, t] \times K} h(z, f(s)) \mathbf{1}_{\left\{M_{h}(z) \leq \rho\right\}}\left[g_{k}(s, z)-g(s, z)\right] v(\mathrm{~d} z) \mathrm{d} s
$$

and

$$
T_{5, \rho}^{k}(t)=\int_{[0, t] \times K} h(z, f(s)) \mathbf{1}_{\left\{M_{h}(z)>\rho\right\}}\left[g_{k}(s, z)-g(s, z)\right] v(\mathrm{~d} z) \mathrm{d} s .
$$

From Lemma 3.2, for every $\delta>0$,

$$
\sup _{k}\left\|T_{5, \rho}^{k}\right\|_{*, T} \leq\left(1+\|f\|_{*, T}\right)\left(2 c\left(\delta, n, M_{h}\right) T \int_{\mathbb{X}} M_{h}(z) \mathbf{1}_{\left\{M_{h}(z)>\rho\right\}} v(\mathrm{~d} z)+2 \delta\right) .
$$

Choose $\delta>0$ and $\rho>0$ such that the right-hand side of the above expression is bounded by $\varepsilon$. A minor modification of [1, Lemma 2.8] shows (see [3, Appendix A.6]) that for every $\rho>0, T_{4, \rho}^{k}(t) \rightarrow 0$ as $k \rightarrow \infty$. Combining the above estimates, we have for every $t \in[0, T]$, $\lim \sup _{k \rightarrow \infty}\left\|T_{2}^{k}(t)\right\| \leq 2 \varepsilon$. Since $\varepsilon>0$ is arbitrary, the above implies that for every $t \in[0, T]$, $T_{2}^{k}(t) \rightarrow 0$ as $k \rightarrow \infty$. Thus, we have shown that the expression on the left-hand side of (5.1) converges to 0 as $k \rightarrow \infty$, which proves the result. 
Proof of Proposition 5.1. Let $\xi_{k}=\xi^{g_{k}}, \xi=\xi^{g}$. We first argue that $\left\{\xi_{k}\right\}_{k \geq 1}$ is precompact in $C\left([0, T]: \mathbb{R}_{+}^{d}\right)$. Note that

$$
\left\|\xi_{k}(t)\right\| \leq \int_{\mathbb{X}_{t}}\left(1+\left\|\xi_{k}(s)\right\|\right) M_{h}(z) g_{k}(s, z) v(\mathrm{~d} z) \mathrm{d} s, \quad t \in[0, T] .
$$

From Lemma 3.2 it follows that, for any $\delta>0$,

$$
\sup _{\psi \in S^{n}} \int_{\mathbb{X}_{T}} M_{h}(z) \psi(s, z) v(\mathrm{~d} z) \mathrm{d} s \leq c\left(\delta, n, M_{h}\right) T \int_{\mathbb{X}} M_{h}(z) v(\mathrm{~d} z)+\delta .
$$

An application of Gronwall's lemma now shows that

$$
\sup _{k \geq 1}\left(1+\left\|\xi_{k}\right\|_{*, T}\right)=\kappa_{1}<\infty
$$

Next, for $0 \leq s \leq t \leq T$ and $\delta>0$,

$$
\begin{aligned}
\left\|\xi_{k}(t)-\xi_{k}(s)\right\| & \leq \int_{(s, t] \times \mathbb{X}}\left\|h\left(z, \xi_{k}(u)\right)\right\| g_{k}(u, z) v(\mathrm{~d} z) \mathrm{d} u \\
& \leq \kappa_{1} \int_{(s, t] \times \mathbb{X}} M_{h}(z) g_{k}(u, z) v(\mathrm{~d} z) \mathrm{d} u \\
& \leq \kappa_{1}\left(c\left(\delta, n, M_{h}\right)(t-s) \int_{\mathbb{X}} M_{h}(z) v(\mathrm{~d} z)+\delta\right) .
\end{aligned}
$$

This shows that $\left\{\xi_{k}\right\}_{k \geq 1}$ is equicontinuous which together with (5.4) proves the desired precompactness. Suppose that $\xi_{k}$ converges along a subsequence to $\bar{\xi}$. From Lemma 5.1, along this subsequence for every $t \in[0, T]$ as $k \rightarrow \infty$,

$$
\int_{\mathbb{X}_{t}} h\left(z, \xi_{k}(s)\right) g_{k}(s, z) v(\mathrm{~d} z) \mathrm{d} s \rightarrow \int_{\mathbb{X}_{t}} h(z, \bar{\xi}(s)) g(s, z) v(\mathrm{~d} z) \mathrm{d} s .
$$

Combining this with the fact that $\xi_{k}$ solves

$$
\xi_{k}(t)=\int_{\mathbb{X}_{t}} h\left(z, \xi_{k}(s)\right) g_{k}(s, z) v(\mathrm{~d} z) \mathrm{d} s, \quad t \in[0, T]
$$

for every $k \geq 1$, and that $\xi_{k}$ converges along the chosen subsequence to $\bar{\xi}$, we have

$$
\bar{\xi}(t)=\int_{\mathbb{X}_{t}} h(z, \bar{\xi}(s)) g(s, z) \nu(\mathrm{d} z) \mathrm{d} s, \quad t \in[0, T] .
$$

By the unique solvability of the above equation and the definition of $\xi$, we now see that $\bar{\xi}=\xi$.

\subsection{Verification of Condition 4.1(b)}

The following is the main result of this section.

Proposition 5.2. Let $n \in \mathbb{N}$ and let $\varphi_{\varepsilon}, \varphi \in \tilde{U}^{n}$ be such that $\varphi_{\varepsilon}$ converges in distribution to $\varphi$ as $\varepsilon \rightarrow 0$. Let $\left\{g^{\varepsilon}\right\}_{\varepsilon>0}$ be as in Condition 2.1 and $g^{0}$ be as introduced at the beginning of Section 5. Then $g^{\varepsilon}\left(\varepsilon N^{\varepsilon^{-1}} \varphi_{\varepsilon}\right) \stackrel{\mathrm{D}}{\rightarrow} g^{0}\left(\nu_{T}^{\varphi}\right)$. 
Proof. Let $\tilde{\varphi}_{\varepsilon}=1 / \varphi_{\varepsilon}$, and recall that $\varphi_{\varepsilon} \in \tilde{U}^{n}$ means that $\varphi_{\varepsilon}=1$ for some compact set in $\mathbb{X}$ and bounded above and below away from 0 on the compact set. Then it is easy to check (see [10, Theorem III.3.24] and also [4, Lemma 2.3]) that

$$
\varepsilon_{t}^{\varepsilon}=\exp \left\{\int_{(0, t] \times \mathbb{X} \times\left[0, \varepsilon^{-1} \varphi_{\varepsilon}\right]} \log \left(\tilde{\varphi_{\varepsilon}}\right) \mathrm{d} \bar{N}+\int_{(0, t] \times \mathbb{X} \times\left[0, \varepsilon^{-1} \varphi_{\varepsilon}\right]}\left(-\tilde{\varphi}_{\varepsilon}+1\right) \mathrm{d} \bar{\nu}_{T}\right\}
$$

is an $\left\{\overline{\mathcal{F}}_{t}\right\}$-martingale and, consequently,

$$
\mathbb{Q}_{T}^{\varepsilon}(G)=\int_{G} \varepsilon_{T}^{\varepsilon}(\tilde{\varphi}) \mathrm{d} \overline{\mathbb{P}} \quad \text { for } G \in \mathscr{B}(\overline{\mathbb{M}})
$$

defines a probability measure on $\overline{\mathrm{M}}$. Furthermore, $\overline{\mathbb{P}}$ and $\mathbb{Q}_{T}^{\varepsilon}$ are mutually absolutely continuous and it can be verified that under $\mathbb{Q}_{T}^{\varepsilon}, \varepsilon N^{\varepsilon^{-1} \varphi_{\varepsilon}}$ has the same law as that of $\varepsilon N^{\varepsilon^{-1}}$ under $\overline{\mathbb{P}}$. Thus, from Condition 2.1 it follows that $\bar{X}^{\varepsilon}=g^{\varepsilon}\left(\varepsilon N^{\varepsilon^{-1}} \varphi_{\varepsilon}\right)$ is $\mathbb{Q}_{T}^{\varepsilon}$ almost surely (a.s.) (and hence $\overline{\mathbb{P}}$ a.s.) the unique solution of

$$
\bar{X}^{\varepsilon}(t)=\varepsilon \int_{\mathbb{X}_{t}} H_{\varepsilon}\left(\varepsilon^{-1}(t-s), z, \varepsilon^{-1} \bar{X}^{\varepsilon}(s-)\right) N^{\varepsilon^{-1} \varphi_{\varepsilon}}(\mathrm{d} s \mathrm{~d} z), \quad t \in[0, T] .
$$

Also note that $\bar{X}^{0}=g^{0}\left(v_{T}^{\varphi}\right)$ solves the integral equation

$$
\bar{X}^{0}(t)=\int_{\mathbb{X}_{t}} h\left(z, \bar{X}^{0}(s)\right) \varphi(s, z) v(\mathrm{~d} z) \mathrm{d} s, \quad t \in[0, T] .
$$

In order to prove the result we need to show that $\bar{X}^{\varepsilon}$ converges in distribution to $\bar{X}^{0}$. We start by showing that $\left\{\bar{X}^{\varepsilon}\right\}_{\varepsilon>0}$ is tight. Note that, for $t \in[0, T]$,

$$
\begin{aligned}
\overline{\mathbb{E}}\left\|\bar{X}^{\varepsilon}\right\|_{*, t} \leq & \overline{\mathbb{E}} \int_{\mathbb{X}_{t}}\left\|\bar{H}\left(\varepsilon^{-1}(t-s), z, \bar{X}^{\varepsilon}(s)\right)\right\| \varphi_{\varepsilon}(s, z) v(\mathrm{~d} z) \mathrm{d} s \\
& +\overline{\mathbb{E}} \int_{\mathbb{X}_{t}}\left\|R_{\varepsilon}\left(\varepsilon^{-1}(t-s), z, \bar{X}^{\varepsilon}(s)\right)\right\| \varphi_{\varepsilon}(s, z) v(\mathrm{~d} z) \mathrm{d} s \\
= & T_{1}^{\varepsilon}(t)+T_{2}^{\varepsilon}(t) .
\end{aligned}
$$

Using the monotonicity of $\bar{H}$, we see that

$$
T_{1}^{\varepsilon}(t) \leq \overline{\mathbb{E}} \int_{\mathbb{X}_{t}}\left\|h\left(z, \bar{X}^{\varepsilon}(s)\right)\right\| \varphi_{\varepsilon}(s, z) v(\mathrm{~d} z) \mathrm{d} s \leq \overline{\mathbb{E}} \int_{\mathbb{X}_{t}}\left(1+\left\|\bar{X}^{\varepsilon}\right\|_{*, s}\right) M_{h}(z) \varphi_{\varepsilon}(s, z) v(\mathrm{~d} z) \mathrm{d} s .
$$

Using Lemma 3.2 we now have, for every $\delta>0$,

$$
T_{1}^{\varepsilon}(t) \leq c\left(\delta, n, M_{h}\right)\left(\int_{\mathbb{X}} M_{h}(z) v(\mathrm{~d} z)\right) \int_{0}^{t}\left(1+\overline{\mathbb{E}}\left\|\bar{X}^{\varepsilon}\right\|_{*, s}\right) \mathrm{d} s+\delta\left(1+\overline{\mathbb{E}}\left\|\bar{X}^{\varepsilon}\right\|_{*, t}\right) .
$$

Another application of Lemma 3.2 shows that, for each fixed $\delta>0$,

$$
\begin{aligned}
T_{2}^{\varepsilon}(t) & \leq \overline{\mathbb{E}} \int_{\mathbb{X}_{t}} \varsigma_{\varepsilon}(z)\left(1+\overline{\mathbb{E}}\left\|\bar{X}^{\varepsilon}\right\|_{*, s}\right) \varphi_{\varepsilon}(s, z) \nu(\mathrm{d} z) \mathrm{d} s \\
& \leq c(\delta, n, \varsigma) \int_{\mathbb{X}} \varsigma_{\varepsilon}(z) \nu(\mathrm{d} z) \int_{0}^{t}\left(1+\overline{\mathbb{E}}\left\|\bar{X}^{\varepsilon}\right\|_{*, s}\right) \mathrm{d} s+\delta\left(1+\overline{\mathbb{E}}\left\|\bar{X}^{\varepsilon}\right\|_{*, t}\right) .
\end{aligned}
$$


Combining the above estimates on $T_{1}^{\varepsilon}$ and $T_{2}^{\varepsilon}$ and choosing $\delta$ sufficiently small, we have, by an application of Gronwall's lemma,

$$
\sup _{\varepsilon>0} \overline{\mathbb{E}}\left\|\bar{X}^{\varepsilon}\right\|_{*, T}=\kappa_{1}<\infty .
$$

In order to prove the tightness of $\left\{\bar{X}^{\varepsilon}\right\}_{\varepsilon>0}$ we will first establish the tightness of the following closely related collection $\left\{\tilde{X}^{\varepsilon}\right\}_{\varepsilon>0}$ of $C\left([0, T]: \mathbb{R}^{d}\right)$-valued random variables:

$$
\tilde{X}^{\varepsilon}(t)=\int_{\mathbb{X}_{t}} h\left(z, \bar{X}^{\varepsilon}(s)\right) \varphi_{\varepsilon}(s, z) v(\mathrm{~d} z) \mathrm{d} s, \quad t \in[0, T], \varepsilon>0 .
$$

We first observe that

$$
\left\|\tilde{X}^{\varepsilon}\right\|_{*, T} \leq\left(1+\left\|\bar{X}^{\varepsilon}\right\|_{*, T}\right) \int_{\mathbb{X}_{T}} M_{h}(z) \varphi_{\varepsilon}(s, z) v(\mathrm{~d} z) \mathrm{d} s .
$$

Combining the above estimate with (5.3) and (5.6), we see that $\sup _{\varepsilon>0} \overline{\mathbb{E}}\left\|\tilde{X}^{\varepsilon}\right\|_{*, T}<\infty$. Also, for $0 \leq s \leq t \leq T$ and $\delta>0$,

$$
\begin{aligned}
\left\|\tilde{X}^{\varepsilon}(t)-\tilde{X}^{\varepsilon}(s)\right\| & \leq \int_{(s, t] \times \mathbb{X}}\left\|h\left(z, \bar{X}^{\varepsilon}(u)\right)\right\| \varphi_{\varepsilon}(u, z) v(\mathrm{~d} z) \mathrm{d} u \\
& \leq\left(1+\left\|\bar{X}^{\varepsilon}\right\|_{*, T}\right)\left(c\left(\delta, n, M_{h}\right)(t-s) \int_{\mathbb{X}} M_{h}(z) v(\mathrm{~d} z)+\delta\right) .
\end{aligned}
$$

Let $\kappa_{2}(\delta)=c\left(\delta, n, M_{h}\right) \int_{\mathbb{X}} M_{h}(z) v(\mathrm{~d} z)$ and consider

$$
\begin{aligned}
A_{\alpha}=\left\{x \in C\left([0, T]: \mathbb{R}^{d}\right):\|x\|_{*, T} \leq \alpha,\right. \\
\left.\quad \text { and for every } \delta>0,\|x(t)-x(s)\| \leq \alpha\left(\kappa_{2}(\delta)(t-s)+\delta\right)\right\} .
\end{aligned}
$$

It is easy to check that for every $\alpha>0, A_{\alpha}$ is a compact subset of $C\left([0, T]: \mathbb{R}^{d}\right)$. Also, from the above estimates, $\sup _{\varepsilon} \overline{\mathbb{P}}\left(\tilde{X}^{\varepsilon} \in A_{\alpha}^{c}\right) \rightarrow 0$ as $\alpha \rightarrow \infty$. This proves the tightness of $\left\{\tilde{X}^{\varepsilon}\right\}_{\varepsilon>0}$. Next, let for $\varepsilon>0$,

$$
\bar{Y}^{\varepsilon}(t)=\varepsilon \int_{\mathbb{X}_{t}} h\left(z, \bar{X}^{\varepsilon}(s-)\right) N^{\varepsilon^{-1} \varphi_{\varepsilon}}(\mathrm{d} s \mathrm{~d} z), \quad t \in[0, T] .
$$

Then, for $t \in[0, T]$,

$$
\bar{Y}^{\varepsilon}(t)-\bar{X}^{\varepsilon}(t)=\varepsilon \int_{\mathbb{X}_{t}}\left[h\left(z, \bar{X}^{\varepsilon}(s-)\right)-\bar{H}\left(\varepsilon^{-1}(t-s), z, \bar{X}^{\varepsilon}(s-)\right)\right] N^{\varepsilon^{-1} \varphi_{\varepsilon}}(\mathrm{d} s \mathrm{~d} z)+\mathcal{R}_{1}^{\varepsilon}(t),
$$

where

$$
\begin{aligned}
\left\|\mathcal{R}_{1}^{\varepsilon}\right\|_{*, T} & \leq \varepsilon \sup _{t \in[0, T]} \int_{\mathbb{X}_{t}}\left\|R_{\varepsilon}\left(\varepsilon^{-1}(t-s), z, \bar{X}^{\varepsilon}(s-)\right)\right\| N^{\varepsilon^{-1} \varphi_{\varepsilon}}(\mathrm{d} s \mathrm{~d} z) \\
& \leq \varepsilon \int_{\mathbb{X}_{T}} \varsigma_{\varepsilon}(z)\left(\left\|\bar{X}^{\varepsilon}(s-)\right\|+1\right) N^{\varepsilon^{-1} \varphi_{\varepsilon}}(\mathrm{d} s \mathrm{~d} z) .
\end{aligned}
$$

Thus, for every $\delta>0$,

$$
\begin{aligned}
\overline{\mathbb{E}}\left\|\mathcal{R}_{1}^{\varepsilon}\right\|_{*, T} & \leq \overline{\mathbb{E}}\left(\left(\left\|\bar{X}^{\varepsilon}\right\|_{*, T}+1\right) \int_{\mathbb{X}_{T}} \varsigma_{\varepsilon}(z) \varphi_{\varepsilon}(s, z) v(\mathrm{~d} z) \mathrm{d} s\right) \\
& \leq\left(\overline{\mathbb{E}}\left\|\bar{X}^{\varepsilon}\right\|_{*, T}+1\right)\left[c(\delta, n, \varsigma) T \int_{\mathbb{X}} \varsigma_{\varepsilon}(z) v(\mathrm{~d} z)+\delta\right]
\end{aligned}
$$


Since $\int_{\mathbb{X}} \zeta_{\varepsilon}(z) \nu(\mathrm{d} z)$ converges to 0 as $\varepsilon \rightarrow 0$, we have

$$
\mathcal{R}_{1}^{\varepsilon} \text { converges to } 0 \text { in probability in } D\left([0, T]: \mathbb{R}^{d}\right) \text {. }
$$

Next, denoting the first term on the right-hand side of $(5.8)$ as $f_{1}^{\varepsilon}(t)$, we have, for $t_{0} \in[0, T]$,

$$
\begin{aligned}
\overline{\mathbb{E}}\left\|\delta_{1}^{\varepsilon}\right\|_{*, t_{0}} & \leq \varepsilon \overline{\mathbb{E}} \int_{\mathbb{X}_{t_{0}}}\left\|h\left(z, \bar{X}^{\varepsilon}(s-)\right)\right\| N^{\varepsilon^{-1} \varphi_{\varepsilon}}(\mathrm{d} s \mathrm{~d} z) \\
& \leq \overline{\mathbb{E}}\left(\left(\left\|\bar{X}^{\varepsilon}\right\|_{*, T}+1\right) \int_{\mathbb{X}_{t_{0}}} M_{h}(z) \varphi_{\varepsilon}(s, z) v(\mathrm{~d} z) \mathrm{d} s\right) \\
& \leq\left(\overline{\mathbb{E}}\left\|\bar{X}^{\varepsilon}\right\|_{*, T}+1\right)\left[c\left(\delta, n, M_{h}\right) t_{0} \int_{\mathbb{X}} M_{h}(z) v(\mathrm{~d} z)+\delta\right] .
\end{aligned}
$$

Thus, for some $\kappa_{1} \in(0, \infty)$, we have, for every $\delta>0$,

$$
\sup _{\varepsilon>0} \overline{\mathbb{E}}\left\|\delta_{1}^{\varepsilon}\right\|_{*, t_{0}} \leq \kappa_{1}\left(t_{0} c\left(\delta, n, M_{h}\right)+\delta\right) .
$$

Now we consider the interval $\left(t_{0}, T\right]$. Note that, for any $v \in\left(0, t_{0}\right)$,

$$
\sup _{t \in\left(t_{0}, T\right]}\left\|\delta_{1}^{\varepsilon}(t)\right\| \leq \sup _{t \in\left(t_{0}, T\right]}\left\|\delta_{1}^{\varepsilon}(t-v)\right\|+\sup _{t \in\left(t_{0}, T\right]}\left\|\delta_{2, v}^{\varepsilon}(t)\right\|
$$

where

$$
s_{2, v}^{\varepsilon}(t)=\varepsilon \int_{(t-v, t] \times \mathbb{X}}\left[h\left(z, \bar{X}^{\varepsilon}(s-)\right)-\bar{H}\left(\varepsilon^{-1}(t-s), z, \bar{X}^{\varepsilon}(s-)\right)\right] N^{\varepsilon^{-1} \varphi_{\varepsilon}}(\mathrm{d} s \mathrm{~d} z) .
$$

Using the monotonicity of $\bar{H}$ again, we have, for $\alpha>0$,

$$
\begin{aligned}
\sup _{t \in\left(t_{0}, T\right]}\left\|f_{1}^{\varepsilon}(t-v)\right\| & \leq \varepsilon \int_{\mathbb{X}_{T}}\left\|h\left(z, \bar{X}^{\varepsilon}(s-)\right)-\bar{H}\left(\varepsilon^{-1} v, z, \bar{X}^{\varepsilon}(s-)\right)\right\| N^{\varepsilon^{-1} \varphi_{\varepsilon}}(\mathrm{d} s \mathrm{~d} z) \\
& =\mathcal{R}_{2, \alpha}^{\varepsilon}+\mathcal{R}_{3, \alpha}^{\varepsilon},
\end{aligned}
$$

where

$$
\begin{aligned}
& \mathcal{R}_{2, \alpha}^{\varepsilon}=\varepsilon 1_{B_{\alpha}^{\varepsilon}} \int_{\mathbb{X}_{T}}\left\|h\left(z, \bar{X}^{\varepsilon}(s-)\right)-\bar{H}\left(\varepsilon^{-1} v, z, \bar{X}^{\varepsilon}(s-)\right)\right\| N^{\varepsilon^{-1}} \varphi_{\varepsilon}(\mathrm{d} s \mathrm{~d} z), \\
& \mathcal{R}_{3, \alpha}^{\varepsilon}=\varepsilon 1_{\left(B_{\alpha}^{\varepsilon}\right)^{c}} \int_{\mathbb{X}_{T}}\left\|h\left(z, \bar{X}^{\varepsilon}(s-)\right)-\bar{H}\left(\varepsilon^{-1} v, z, \bar{X}^{\varepsilon}(s-)\right)\right\| N^{\varepsilon^{-1}} \varphi_{\varepsilon}(\mathrm{d} s \mathrm{~d} z),
\end{aligned}
$$

and $B_{\alpha}^{\varepsilon}=\left\{\omega:\left\|\bar{X}^{\varepsilon}\right\|_{*, T} \leq \alpha\right\}$. For $\alpha>0$, let $\varpi_{\alpha}: \mathbb{R}_{+} \times \mathbb{X} \rightarrow \mathbb{R}_{+}$be defined as

$$
\varpi_{\alpha}(r, z)=\sup _{\|x\| \leq \alpha}\|h(z, x)-\bar{H}(r, z, x)\|, \quad(r, z) \in \mathbb{R}_{+} \times \mathbb{X} .
$$

Then, from Condition 2.2(e), for all $(\alpha, z) \in \mathbb{R}_{+} \times \mathbb{X}, \varpi_{\alpha}(r, z) \rightarrow 0$ as $r \rightarrow \infty$. Also, since $\varpi_{\alpha}(r, z) \leq M_{h}(z)(1+\alpha)$ and $M_{h} \in \mathcal{L}^{1}(\nu)$, we have $\int_{\mathbb{X}} \varpi_{\alpha}(r, z) v(\mathrm{~d} z) \rightarrow 0$ as $r \rightarrow \infty$. Now, for every $\delta>0$,

$$
\begin{aligned}
\overline{\mathbb{E}} \mathcal{R}_{2, \alpha}^{\varepsilon} & \leq \overline{\mathbb{E}} \int_{\mathbb{X}_{T}} \varpi_{\alpha}\left(\varepsilon^{-1} v, z\right) \varphi_{\varepsilon}(s, z) v(\mathrm{~d} z) \mathrm{d} s \\
& \leq T c\left(\delta, n, M_{h}\right) \int_{\mathbb{X}} \varpi_{\alpha}\left(\varepsilon^{-1} v, z\right) v(\mathrm{~d} z)+\delta(1+\alpha) .
\end{aligned}
$$

Thus, $\mathcal{R}_{2, \alpha}^{\varepsilon} \rightarrow 0$ as $\varepsilon \rightarrow 0$ for every $\alpha>0$. 
Next, from Markov's inequality and (5.6), for $\eta>0$,

$$
\sup _{\varepsilon>0} \overline{\mathbb{P}}\left(\mathcal{R}_{3, \alpha}^{\varepsilon}>\eta\right) \leq \sup _{\varepsilon>0} \overline{\mathbb{P}}\left(\left(B_{\alpha}^{\varepsilon}\right)^{c}\right) \leq \sup _{\varepsilon>0} \overline{\mathbb{P}}\left(\left\|\bar{X}^{\varepsilon}\right\|_{*, T}>\alpha\right) \leq \frac{\kappa_{1}}{\alpha} .
$$

Using the above two observations in (5.12), we have for every $t_{0} \in(0, T)$ and $v \in\left(0, t_{0}\right)$,

$$
\sup _{t \in\left(t_{0}, T\right]}\left\|f_{1}^{\varepsilon}(t-v)\right\| \stackrel{\mathbb{P}}{\rightarrow} 0 \quad \text { as } \varepsilon \rightarrow 0
$$

where $\stackrel{\mathbb{P}}{\rightarrow}$ ' denotes convergence in probability. We now consider $\delta_{2, v}^{\varepsilon}$. Using monotonicity of $H$,

$$
\begin{aligned}
\sup _{t \in\left(t_{0}, T\right]}\left\|f_{2, v}^{\varepsilon}(t)\right\| \leq & \sup _{t \in\left(t_{0}, T\right]} \varepsilon\left\|\int_{(t-v, t] \times \mathbb{X}} h\left(z, \bar{X}^{\varepsilon}(s-)\right) N^{\varepsilon^{-1} \varphi_{\varepsilon}}(\mathrm{d} s \mathrm{~d} z)\right\| \\
\leq & \sup _{t \in\left(t_{0}, T\right]} \varepsilon\left\|\int_{(t-v, t] \times \mathbb{X}} h\left(z, \bar{X}^{\varepsilon}(s-)\right) \tilde{N}^{\varepsilon^{-1} \varphi_{\varepsilon}}(\mathrm{d} s \mathrm{~d} z)\right\| \\
& +\sup _{t \in\left(t_{0}, T\right]}\left\|\int_{(t-v, t] \times \mathbb{X}} h\left(z, \bar{X}^{\varepsilon}(s-)\right) \varphi_{\varepsilon}(s, z) v(\mathrm{~d} z) \mathrm{d} s\right\| \\
& =\mathcal{R}_{4}^{\varepsilon}\left(t_{0}, v\right)+\mathcal{R}_{5}^{\varepsilon}\left(t_{0}, v\right),
\end{aligned}
$$

where $\tilde{N}^{\varepsilon^{-1} \varphi_{\varepsilon}}(\mathrm{d} s \mathrm{~d} z)=N^{\varepsilon^{-1}} \varphi_{\varepsilon}(\mathrm{d} s \mathrm{~d} z)-\varepsilon^{-1} \varphi_{\varepsilon}(s, z) \nu_{T}(\mathrm{~d} s \mathrm{~d} z)$, the compensated version of $N^{\varepsilon^{-1}} \varphi_{\varepsilon}$. For $\eta>0$ and $\alpha>0$,

$$
\overline{\mathbb{P}}\left(\mathcal{R}_{5}^{\varepsilon}\left(t_{0}, v\right)>\eta\right) \leq \overline{\mathbb{P}}\left(\mathcal{R}_{5}^{\varepsilon}\left(t_{0}, v\right)>\eta ; B_{\alpha}^{\varepsilon}\right)+\overline{\mathbb{P}}\left(\left(B_{\alpha}^{\varepsilon}\right)^{c}\right)
$$

Also,

$$
\begin{aligned}
\mathcal{R}_{5}^{\varepsilon}\left(t_{0}, v\right) \mathbf{1}_{B_{\alpha}^{\varepsilon}} & \leq(1+\alpha) \sup _{t \in\left(t_{0}, T\right]} \int_{(t-v, t] \times \mathbb{X}} M_{h}(z) \varphi_{\varepsilon}(s, z) v(\mathrm{~d} z) \mathrm{d} s \\
& \leq(1+\alpha)\left[v c\left(\delta, n, M_{h}\right) \int_{\mathbb{X}} M_{h}(z) v(\mathrm{~d} z)+\delta\right] .
\end{aligned}
$$

Combining the above two estimates and using (5.13) once again, we have for every $t_{0} \in(0, T)$ and $\eta>0$,

$$
\sup _{\varepsilon>0} \overline{\mathbb{P}}\left(\mathcal{R}_{5}^{\varepsilon}\left(t_{0}, v\right)>\eta\right) \rightarrow 0 \quad \text { as } v \rightarrow 0 .
$$

We now consider $\mathcal{R}_{4}^{\varepsilon}\left(t_{0}, v\right)$. We have

$$
\mathcal{R}_{4}^{\varepsilon}\left(t_{0}, v\right) \leq 2 \varepsilon \sup _{0 \leq t \leq T}\left\|\int_{\mathbb{X}_{t}} h\left(z, \bar{X}^{\varepsilon}(s-)\right) \tilde{N}^{\varepsilon^{-1}} \varphi_{\varepsilon}(\mathrm{d} s \mathrm{~d} z)\right\|=\tilde{\mathcal{R}}_{4}^{\varepsilon} .
$$

Let $\tau_{\alpha}^{\varepsilon}=\inf \left\{t \in[0, T]:\left\|\bar{X}^{\varepsilon}(t)\right\|>\alpha\right\}$, where the infimum is taken to be $T$ if the set is empty. Let

$$
\mathcal{R}_{6, \alpha}^{\varepsilon}=2 \varepsilon \sup _{0 \leq t \leq T}\left\|\int_{\left(0, t \wedge \tau_{\alpha}^{\varepsilon}\right] \times \mathbb{X}} h\left(z, \bar{X}^{\varepsilon}(s-)\right) \tilde{N}^{\varepsilon^{-1} \varphi_{\varepsilon}}(\mathrm{d} s \mathrm{~d} z)\right\| .
$$

Then, from (5.13), for $\eta>0$,

$$
\overline{\mathbb{P}}\left(\mathcal{R}_{4}^{\varepsilon}\left(t_{0}, v\right)>\eta\right) \leq \overline{\mathbb{P}}\left(\tilde{\mathcal{R}}_{4}^{\varepsilon}>\eta\right) \leq \overline{\mathbb{P}}\left(\mathcal{R}_{6, \alpha}^{\varepsilon}>\eta\right)+\overline{\mathbb{P}}\left(\left(B_{\alpha}^{\varepsilon}\right)^{c}\right) \leq \overline{\mathbb{P}}\left(\mathcal{R}_{6, \alpha}^{\varepsilon}>\eta\right)+\frac{\kappa_{1}}{\alpha}
$$


For $r>0$, write $\mathcal{R}_{6, \alpha}^{\varepsilon}=\mathcal{R}_{7, \alpha}^{\varepsilon, r}+\mathcal{R}_{8, \alpha}^{\varepsilon, r}$, where

$$
\begin{aligned}
& \mathcal{R}_{7, \alpha}^{\varepsilon, r}=2 \varepsilon \sup _{0 \leq t \leq T} \| \int_{\left(0, t \wedge \tau_{\alpha}^{\varepsilon}\right] \times \mathbb{X}} h\left(z, \bar{X}^{\varepsilon}(s-)\right) \mathbf{1}_{\left\{M_{h}(z) \leq r\right\}} \tilde{N}^{\varepsilon^{-1} \varphi_{\varepsilon}(\mathrm{d} s \mathrm{~d} z) \|,} \\
& \mathcal{R}_{8, \alpha}^{\varepsilon, r}=2 \varepsilon \sup _{0 \leq t \leq T}\left\|\int_{\left(0, t \wedge \tau_{\alpha}^{\varepsilon}\right] \times \mathbb{X}} h\left(z, \bar{X}^{\varepsilon}(s-)\right) \mathbf{1}_{\left\{M_{h}(z)>r\right\}} \tilde{N}^{\varepsilon^{-1} \varphi_{\varepsilon}}(\mathrm{d} s \mathrm{~d} z)\right\| .
\end{aligned}
$$

By standard martingale inequalities, for some $\kappa_{2} \in(0, \infty)$ (independent of $\left.\alpha, \varepsilon, r\right)$,

$$
\begin{aligned}
\overline{\mathbb{E}}\left(\mathcal{R}_{7, \alpha}^{\varepsilon, r}\right)^{2} & \leq \kappa_{2} r\left(1+\alpha^{2}\right) \varepsilon \overline{\mathbb{E}} \int_{\mathbb{X}_{T}} M_{h}(z) \varphi_{\varepsilon}(s, z) v(\mathrm{~d} z) \mathrm{d} s \\
& \leq \kappa_{2} r\left(1+\alpha^{2}\right) \varepsilon\left(c\left(\delta, n, M_{h}\right) \int_{\mathbb{X}} M_{h}(z) v(\mathrm{~d} z)+\delta\right)
\end{aligned}
$$

Thus, for each $r>0$ and $\alpha>0, \mathcal{R}_{7, \alpha}^{\varepsilon, r} \stackrel{\mathbb{P}}{\rightarrow} 0$ as $\varepsilon \rightarrow 0$. Also, for every $\delta>0$,

$$
\begin{aligned}
\overline{\mathbb{E}}\left(\mathcal{R}_{8, \alpha}^{\varepsilon, r}\right) & \leq 8(1+\alpha) \mathbb{E} \int_{\mathbb{X}_{T}} M_{h}(z) \mathbf{1}_{\left\{M_{h}(z)>r\right\}} \varphi_{\varepsilon}(s, z) v(\mathrm{~d} z) \mathrm{d} s \\
& \leq 8(1+\alpha)\left(c\left(\delta, n, M_{h}\right) \int_{\mathbb{X}} M_{h}(z) \mathbf{1}_{\left\{M_{h}(z)>r\right\}} v(\mathrm{~d} z)+\delta\right) .
\end{aligned}
$$

Since $M_{h} \in \mathcal{L}^{1}(\nu)$, we have for each $\alpha>0, \sup _{\varepsilon>0} \overline{\mathbb{E}}\left(\mathcal{R}_{8, \alpha}^{\varepsilon, r}\right)$ converges to 0 as $r \rightarrow \infty$. Combining the above estimates, for each $\alpha>0$,

$$
\mathcal{R}_{6, \alpha}^{\varepsilon} \stackrel{\mathbb{P}}{\rightarrow} 0 \quad \text { as } \varepsilon \rightarrow 0 .
$$

Using this observation in (5.18), we have for each $t_{0} \in(0, T)$ and $v \in\left(0, t_{0}\right)$,

$$
\mathcal{R}_{4}^{\varepsilon}\left(t_{0}, v\right) \text { and } \tilde{\mathcal{R}}_{4}^{\varepsilon} \stackrel{\mathbb{P}}{\rightarrow} 0 \quad \text { as } \varepsilon \rightarrow 0 .
$$

Combining this with (5.11), (5.14), (5.16), and (5.17), we see that, for every $t_{0} \in(0, T)$,

$$
\sup _{t \in\left(t_{0}, T\right]}\left\|\mathcal{f}_{1}^{\varepsilon}(t)\right\| \stackrel{\mathbb{P}}{\rightarrow} 0 \quad \text { as } \varepsilon \rightarrow 0 .
$$

Thus, from (5.10),

$$
\sup _{t \in[0, T]}\left\|\delta_{1}^{\varepsilon}(t)\right\| \stackrel{\mathbb{P}}{\rightarrow} 0 \quad \text { as } \varepsilon \rightarrow 0 .
$$

Combining this with (5.9) and (5.8), we see that

$$
\bar{Y}^{\varepsilon}-\bar{X}^{\varepsilon} \stackrel{\mathbb{P}}{\rightarrow} 0 \quad \text { in } D\left([0, T]: \mathbb{R}^{d}\right) .
$$

Also, since $\left\|\bar{Y}^{\varepsilon}-\tilde{X}^{\varepsilon}\right\|_{*, T}=\frac{1}{2} \tilde{\mathcal{R}}_{4}^{\varepsilon}$, from (5.19), we have

$$
\bar{Y}^{\varepsilon}-\tilde{X}^{\varepsilon} \stackrel{\mathbb{P}}{\rightarrow} 0 \quad \text { in } D\left([0, T]: \mathbb{R}^{d}\right) .
$$

The above two displays together with the tightness of $\left\{\tilde{X}^{\varepsilon}\right\}_{\varepsilon>0}$ established earlier show that $\left\{\bar{X}^{\varepsilon}\right\}_{\varepsilon>0}$ is tight. Suppose that $\left(\bar{X}^{\varepsilon}, \varphi_{\varepsilon}\right)$ converges weakly along a subsequence to $\left(X^{0}, \tilde{\varphi}\right)$. 
Note that $\varphi$ and $\tilde{\varphi}$ must have the same distribution. Without loss of generality we can assume that convergence is almost sure and it holds along the full sequence. In fact we can assume that $\left(\bar{X}^{\varepsilon}, \tilde{X}^{\varepsilon}, \varphi_{\varepsilon}\right)$ converges a.s. to $\left(X^{0}, X^{0}, \tilde{\varphi}\right)$. Then, from Lemma 5.1, for all $t \in[0, T]$,

$$
\int_{\mathbb{X}_{t}} h\left(z, \bar{X}^{\varepsilon}(s)\right) \varphi_{\varepsilon}(s, z) v(\mathrm{~d} z) \mathrm{d} s \rightarrow \int_{\mathbb{X}_{t}} h\left(z, X^{0}(s)\right) \tilde{\varphi}(s, z) v(\mathrm{~d} z) \mathrm{d} s \quad \text { a.s. }
$$

This, along with (5.7) shows that $X^{0}$ solves

$$
X^{0}(t)=\int_{\mathbb{X}_{t}} h\left(z, X^{0}(s)\right) \tilde{\varphi}(s, z) v(\mathrm{~d} z) \mathrm{d} s, \quad t \in[0, T], \text { a.s. }
$$

Since the above equation has a unique solution and $\tilde{\varphi}$ and $\varphi$ have the same distribution, it follows that $X^{0}$ and $\bar{X}^{0}$ have the same distribution, where $\bar{X}^{0}$ was defined in (5.5). Thus, we have shown that $\bar{X}^{\varepsilon}$ converges in distribution to $\bar{X}^{0}$. The result follows.

\subsection{Completing the Proof of Theorem 2.2}

In view of Theorem 4.1 it suffices to check that $\left\{g^{\varepsilon}, \varepsilon>0\right\}$ introduced in Condition 2.1 and $g^{0}$ introduced at the beginning of Section 5 satisfy Condition 4.1. Condition 4.1(a) is immediate from Proposition 5.1, while Condition 4.1(b) is a consequence of Proposition 5.2. These observations complete the proof.

\section{Acknowledgements}

Amarjit Budhiraja was supported in part by the National Science Foundation (grant nos DMS-1004418, DMS-1016441, and DMS-1305120) and the Army Research Office (grant nos. W911NF-10-1-0158 and W911NF-14-1-0331). Part of this work was carried out when Pierre Nyquist was visiting the University of North Carolina at Chapel Hill. Support and hospitality of the STOR Department at UNC is gratefully acknowledged.

\section{References}

[1] BouÉ, M. AND DuPUIS, P. (1998). A variational representation for certain functionals of Brownian motion. Ann. Prob. 26, 1641-1659.

[2] Brockwell, P. J., Resnick, S. I. And Tweedie, R. L. (1982). Storage processes with general release rule and additive inputs. Adv. Appl. Prob. 14, 392-433.

[3] Budhiraja, A., Chen, J. ANd Dupuis, P. (2013). Large deviations for stochastic partial differential equations driven by a Poisson random measure. Stoch. Process. Appl. 123, 523-560.

[4] Budhiraja, A., Dupuis, P. And Maroulas, V. (2011). Variational representations for continuous time processes. Ann. Inst. H. Poincaré Prob. Statist. 47, 725-747.

[5] Daley, D. J. (1971). The definition of a multi-dimensional generalization of shot noise. J. Appl. Prob. 8, 128-135.

[6] Dembo, A. ANd Zeitouni, O. (1998). Large Deviations Techniques and Applications, 2nd edn. Springer, New York.

[7] Doney, R. A. and O'Brien, G. L. (1991). Loud shot noise. Ann. Appl. Prob. 1, 88-103.

[8] Ganesh, A., Macci, C. And Torrisi, G. L. (2005). Sample path large deviations principles for Poisson shot noise processes, and applications. Electron. J. Prob. 10, 1026-1043.

[9] Huffer, F. W. (1987). Inequalities for the $M / G / \infty$ queue and related shot noise processes. J. Appl. Prob. 24, 978-989.

[10] Jacod, J. and Shiryaev, A. N. (2003). Limit Theorems for Stochastic Processes, 2nd edn. Springer, Berlin.

[11] KlÜPPElberg, C. AND MikosCh, T. (1995). Explosive Poisson shot noise processes with applications to risk reserves. Bernoulli 1, 125-147.

[12] KLÜPPElbERG, C., MikosCh, T. AND SchärF, A. (2003). Regular variation in the mean and stable limits for Poisson shot noise. Bernoulli 9, 467-496.

[13] Lane, J. A. (1984). The central limit theorem for the Poisson shot-noise process. J. Appl. Prob. 21, $287-301$. 
[14] Lund, R. B. (1996). The stability of storage models with shot noise input. J. Appl. Prob. 33, 830-839.

[15] Lund, R., McCormick, W. P. AND XIAO, Y. (2004). Limiting properties of Poisson shot noise processes. J. Appl. Prob. 41, 911-918.

[16] McCormick, W. P. (1997). Extremes for shot noise processes with heavy tailed amplitudes. J. Appl. Prob. 34, 643-656.

[17] Rice, J. (1977). On generalized shot noise. Adv. Appl. Prob. 9, 553-565.

[18] Rice, S. O. (1945). Mathematical analysis of random noise. Bell System Tech. J. 24, 46-156.

[19] Samorodnitsky, G. (1998). Tail behavior of some shot noise processes. In A Practical Guide to Heavy Tails, Birkhäuser, Boston, MA, pp. 473-478.

[20] TorrisI, G. L. (2004). Simulating the ruin probability of risk processes with delay in claim settlement. Stoch. Process. Appl. 112, 225-244. 